\title{
Review of the Literature on the Occurrence of Delirium after Veno-Venous and Veno-Arterial Extracorporeal Membrane Oxygenation: A Systematic Review
}

\author{
Sabina Krupa Dorota Ozga \\ Collegium Medicum, Rzeszow University, Rzeszow, Poland
}

Keywords

Extracorporeal membrane oxygenation · Delirium · Critically ill patients · Systematic review

\begin{abstract}
Introduction: Extracorporeal membrane oxygenation (ECMO) is an extracorporeal gas exchange method which, despite a number of advantages, carries the risk of many complications. ECMO is a modern intensive care method which in many cases is the last resort for the patient. Care and supervision are provided by a multidisciplinary team of specialists: physicians, perfusionists, and nurses. The aim of this review is to analyze the occurrence of delirium in ECMO patients. Methods: Both authors independently extracted data from all included trials and assessed the risk of bias. A systematic review was performed using the protocol of the Cochrane Collaboration Risk of Bias tool. The search was based on PubMed, Web of Science, and Mendeley. Three articles from recent years have been analyzed in this work. Literature selection was made using the PRISMA checklist. The analyzed literature proves how important the topic of delirium is in ECMO therapy. In the case of pharmacotherapy, there are many combinations of drugs that prevent the occurrence of the delirium phenomenon. Results: This work deals with the subject of delirium after ECMO, which is not a common subject in the popular literature. Many of the elements mentioned in the articles analyzed show how important this topic is. The authors place great emphasis on the elements which are not related to pharmacotherapy and the prevention of delirium. For the prevention of delirium after ECMO, a psychological approach to the patient is important. As far as pharmacotherapy is concerned, it is the last element to be taken into account in the prevention of delirium in ECMO patients. An overview of the literature indicates that the subject of nursing care has been omitted; however, there are tools which allow nurses to assess delirium in patients. Conclusion: Delirium
\end{abstract}


in patients undergoing ECMO therapy is a topic that has not been fully described in the literature. This review of the literature shows how important it is to treat a patient with delirium during this therapy and how important it may be to have an early diagnosis of delirium to prevent complications.

\section{Introduction}

This work deals with the subject of delirium after extracorporeal membrane oxygenation (ECMO), which is not a common subject in the popular literature. Many of the elements mentioned in the articles analyzed show how important this topic is. The authors place great emphasis on all elements which are not related to pharmacotherapy and the prevention of delirium. For the prevention of delirium in ECMO patients, a psychological approach to the patient is important. As far as pharmacotherapy is concerned, it is the last element to be taken into account in the prevention of delirium in ECMO patients. An overview of the literature indicates that the subject of nursing care has been omitted; however, there are tools which allow nurses to assess delirium in patients $[1,2]$.

\section{Methods}

A systematic review of the literature published in English was conducted according to the checklist of Preferred Reporting Items for Systematic Reviews and Meta-Analyses (PRISMA) using manual and electronic literature searching strategies. Inclusion and exclusion criteria were established. We systematically searched for relevant studies published prior to December 26, 2018, in Mendeley, PubMed, and Web of Science. The following terms were used for the electronic search: \#ICU nurses, \#extracorporeal membrane oxygenation, \#delirium, \#delirium in ECMO, and \#delirium in extracorporeal membrane oxygenation.

No language restrictions were applied, and the reference lists from all studies included were manually checked to identify other relevant articles. Both authors independently searched and evaluated the quality of the research. The use of a combination of at least two of the accepted keywords has helped to identify 30 articles approved for further stages of analysis. At the next stage, 27 articles were disregarded. Only 3 articles met the ECMO delirium-related criteria (Tables 1, 2).

\section{Results}

\section{Study Selection and Data Extraction}

Both authors independently evaluated the titles and abstracts of all articles retrieved to identify potentially relevant studies. A full-text review was conducted when either reviewer deemed that the abstract warranted further investigation on the basis of our a priori eligibility criteria. A systematic review of the literature published in English was conducted according to the checklist of PRISMA using manual and electronic literature searching strategies. Inclusion and exclusion criteria were established. We systematically searched for relevant studies published prior to December 26, 2018, in Mendeley, PubMed, and Web of Science. The following terms were used for the electronic search: \#ICU nurses, \#extracorporeal membrane oxygenation, \#delirium, \#delirium in ECMO, and \#delirium in extracorporeal membrane oxygenation. 
Table 1. PRISMA for delirium in VV and VA ECMO

\begin{tabular}{llr}
\hline Identification & $\begin{array}{l}\text { Records identified through database } \\
\text { search (based on keywords and full text } \\
\text { availability) }(n=30)\end{array}$ & $\begin{array}{l}\text { Records identified through manual search in journals' } \\
\text { databases (2008-2018 or availability) }(n=30)\end{array}$ \\
\hline Screening & Records screened $(n=26)$ & $\begin{array}{c}\text { Record after duplicates removed }(n=26) \\
\text { Records excluded }(n=3)\end{array}$ \\
\hline Eligibility & $\begin{array}{l}\text { Full-text articles assessed for eligibility } \\
(n=3)\end{array}$ & $\begin{array}{l}\text { Full-text articles excluded }(n=20) \\
\text { Grounds for exclusion: articles not exclusively devoted to } \\
\text { delirium in ECMO, articles about children, abstracts }\end{array}$ \\
\hline Included & \multicolumn{2}{c}{ Studies included in qualitative review $(n=3)$} \\
\hline ECMO, extracorporeal membrane oxygenation; VV, veno-venous; VA, veno-arterial.
\end{tabular}

Table 2. Summary of the studies included in the analysis

\begin{tabular}{llll}
\hline No. Reference & Title of the publication & $\begin{array}{l}\text { Year of } \\
\text { publication }\end{array}$ & Aim of the study \\
\hline $\begin{array}{l}\text { Acevedo-Nuevo, } \\
\text { et al. [3] }\end{array}$ & $\begin{array}{l}\text { The early diagnosis and } \\
\text { management of mixed delirium } \\
\text { in a patient placed on ECMO and } \\
\text { with difficult sedation: A case } \\
\text { report }\end{array}$ & 2018 & $\begin{array}{l}\text { Research } \\
\text { method, } \\
\text { research } \\
\text { tool }\end{array}$ \\
& $\begin{array}{l}\text { The aim of this article was to present the } \\
\text { case of a patient placed on ECMO, who was } \\
\text { diagnosed as having a mixed delirium and } \\
\text { difficult sedation criteria, and outline the } \\
\text { various assessment strategies and the } \\
\text { employed pharmacological and non- } \\
\text { pharmacological management }\end{array}$ \\
\hline $\begin{array}{l}\text { DeGrado, } \\
\text { et al. [4] }\end{array}$ & $\begin{array}{l}\text { Evaluation of sedatives, } \\
\text { analgesics, and neuromuscular } \\
\text { blocking agents in adults } \\
\text { receiving extracorporeal } \\
\text { membrane oxygenation }\end{array}$ & 2017 & $\begin{array}{l}\text { The objective of this study was to } \\
\text { evaluate the use of sedative, analgesic, } \\
\text { and neuromuscular blocking agents in } \\
\text { patients undergoing ECMO support }\end{array}$ \\
\hline $\begin{array}{l}\text { deBacker } \\
\text { et al. [5] }\end{array}$ & $\begin{array}{l}\text { Sedation practice in } \\
\text { extracorporeal membrane } \\
\text { oxygenation-treated patients } \\
\text { with acute respiratory distress } \\
\text { syndrome: a retrospective study }\end{array}$ & & $\begin{array}{l}\text { The objective was to characterize sedation } \\
\text { management in adult patients with severe } \\
\text { respiratory distress syndrome treated with } \\
\text { venovenous ECMO }\end{array}$ \\
\hline
\end{tabular}

ECMO, extracorporeal membrane oxygenation.

No language restrictions were applied, and the reference lists from all studies included were manually checked to identify other relevant articles. Both authors independently searched and evaluated the quality of the research. The use of a combination of at least two of the accepted keywords has helped to identify 30 articles approved for further stages of analysis. At the next stage, 27 articles were disregarded. Only 3 articles met the ECMO delirium-related criteria.

The process of searching and analyzing articles conducted in line with the inclusion criteria shows that there is no research regarding ICU nurses. The final analysis included 3 articles which met the inclusion criteria. 
Krupa and Ozga: Delirium in ECMO Patients

Table 3. Most important factors identified on the basis of the available literature

\begin{tabular}{|c|c|}
\hline eference & Most important factors \\
\hline $\begin{array}{l}\text { Grado } \\
\text { al. [4] }\end{array}$ & 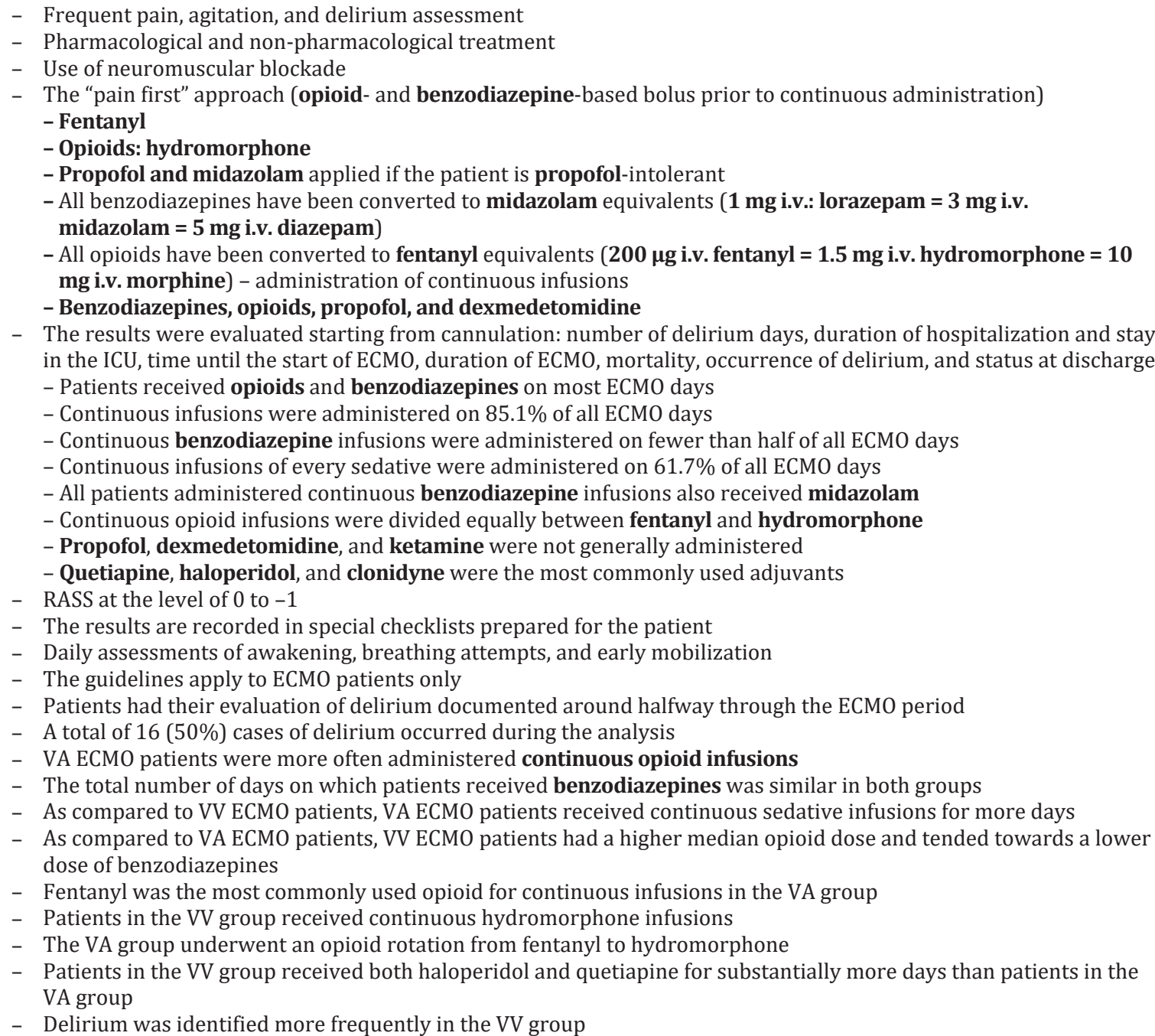 \\
\hline
\end{tabular}

Acevedo- $\quad$ - It is recommended to avoid certain medications (opioids, benzodiazepines, etc.)

Nuevo - Analgosedation

et al. [3] - Preventing sensory deprivation by allowing the use of glasses and hearing aids

- Preventing sleep deprivation; assessment based on the following scales: NRS, CAM-ICU, RASS, BIS, and ESCID

- Monitoring the depth of sedation/agitation with the RASS scale every 4-8 $\mathrm{h}$ in accordance with the unit protocol

- It is recommended that approved tools be used to monitor delirium: Intensive Care Delirium Screening Checklist and Confusion Assessment Method for Intensive Care Units

- Refraining from physical coercion

- Early mobilization

- The patient received fentanyl from the moment of admission until the moment of activation (sitting); midazolam was used for 12 days (from the day of taking up to half-time of ECMO), while remifentanil was only given to the patient on days 2 and 3 of their stay in the ICU (before ECMO); propofol was used from day 5 to 9 and included the time when ECMO therapy was implemented; clonidine was used twice - between days 6 and 7 and from day 11 to 18 when the patient was already connected to the ECMO circuit; cisatracurium was used immediately before connecting the ECMO circuit; treatment with Dexmedetomidine was continued since the introduction of ECMO therapy for the next 4 days; haloperidol administration was required on the day after ECMO implementation for 3 days only in boluses; due to increased pain (according to the ESCID scale), ketamine administration was introduced and continued for 2 days; pentothal was included on day 4 after the start of the therapy and was maintained until the removal of ECMO cannula 
Krupa and Ozga: Delirium in ECMO Patients

Table 3 (continued)

\begin{tabular}{|c|c|}
\hline Reference & Most important factors \\
\hline $\begin{array}{l}\text { deBacker } \\
\text { et al. [5] }\end{array}$ & 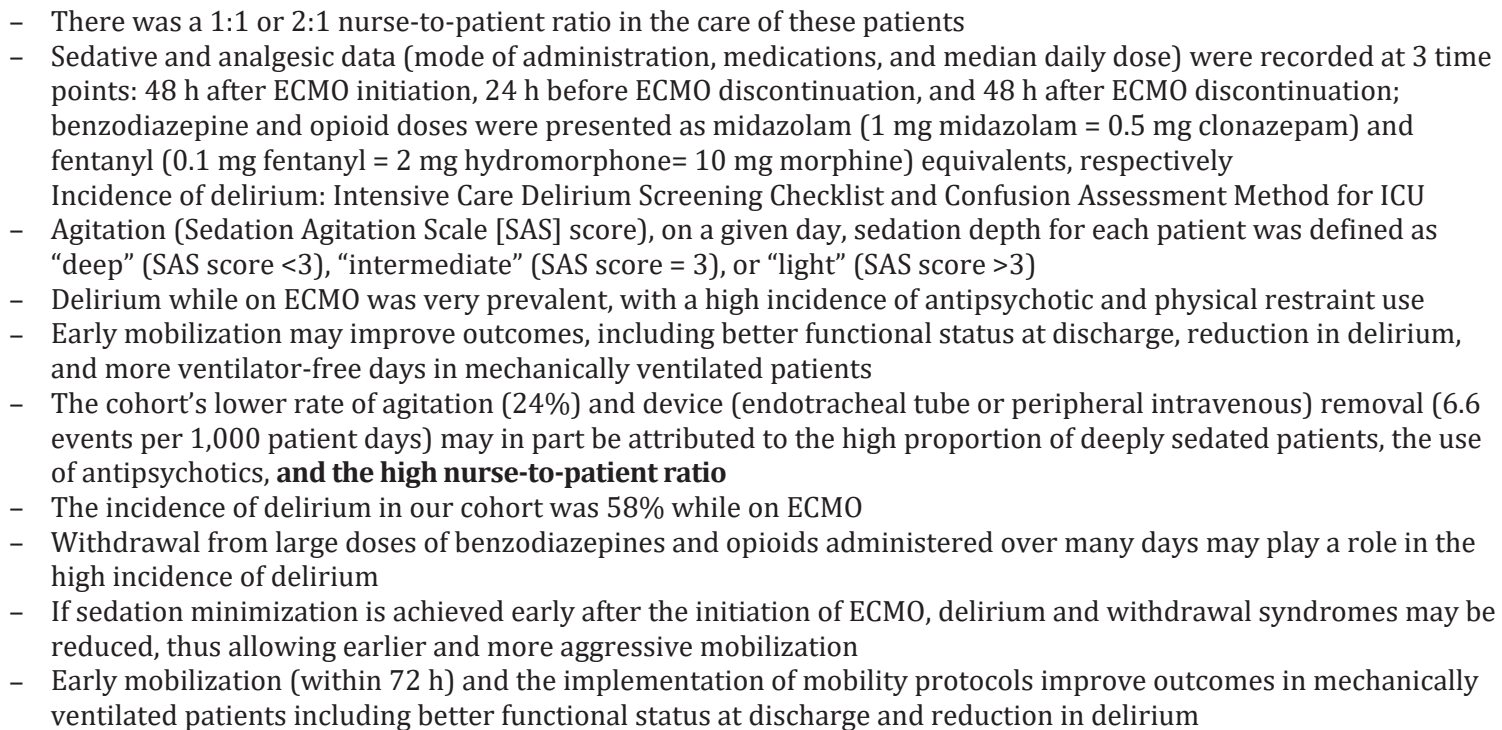 \\
\hline
\end{tabular}

VA, veno-arterial; ECMO, extracorporeal membrane oxygenation; VV, veno-venous; CAM-ICU, Confusion Assessment Method for Intensive Care Units.

Three of the original 30 articles are theoretical papers describing issues analyzed based on the literature, and 5 papers are empirical studies showing the results of research among nurses. The details of the selected methodological aspects are provided in 3 articles.

This thematic analysis resulted in the identification of the most important factors when dealing with delirium in ECMO patients (Table 3) [3-5].

\section{Implications for Clinical Practice}

Conscious sedation and strict monitoring protocols for pain, sedation/agitation, and delirium using validated tools allow an early diagnosis and management of patients. In the case of delirious critically ill patients undergoing ECMO, daily care is challenging and risky due to the highly critical condition of these patients and the risk associated with life-threatening devices. Nursing interventions, such as cognitive stimulation, refraining from the use of physical restraints, reality orientation, or improvement of sleep patterns can prove effective strategies for the management and recovery of critically ill patients with delirium. Early implementation of pharmacological and non-pharmacological measures entails better results at the patient's discharge from the critical care unit.

\section{Conclusions}

The management of mixed delirium in a patient on ECMO and with difficult sedation criteria is challenging for the entire ICU staff. This factor, coupled with other elements, could contribute to a difficult sedation management and mixed delirium, which are handled through a multimodal approach and avoidance of the use of physical restraints. Finally, we can conclude that the multimodal and multidisciplinary approach to the patient using a combi- 
nation of nursing interventions, strict pain, agitation, and delirium monitoring, and pharmacological measures can result in good patient results reaching significant milestones in a relatively short period of time. Failure to diagnose and treat delirium is a serious problem which affects the physical and cognitive judgement of the patient $[6,7]$.

Currently, all articles published on this topic offer a general outline of delirium in ECMO, but they do not address the topic of nursing care provided to delirium patients. These works present many essential conditions that must be met in order to avoid delirium. It is highly important that the patient is mobilized and activated. Long immobilization can cause a fullblown delirium and extend the patient's stay at the ICU. The available literature does not address the topic of nurse participation while caring for a patient with delirium during an ECMO therapy. While the subject of pain and the analysis of risk factors are approached in a very precise manner, the role of the nurse in this respect is not clearly indicated. The authors emphasize the importance of all elements which are not related to pharmacotherapy. For the case of prevention, psychological aspects should be employed: conversation, keeping the patient informed about the activities performed, refraining from raising one's voice, allowing the use of glasses or hearing aids, allowing contact with the family, etc. Furthermore, direct coercion, according to the authors' knowledge, should only be used in very specific cases. Pharmacotherapy should only be used as a last resort, if the patient is dangerous for themselves and people around them $[8,9]$.

\section{Statement of Ethics}

The consent of the bioethical commission was not needed to conduct a literature review due to the type of article. The authors state that the literature review does not affect the privacy of patients in any way, as it only concerns the analysis of research results of other researchers.

\section{Disclosure Statement}

The authors have no conflicts of interest to declare.

\section{Author Contributions}

Both authors made the same contribution to writing the paper and collaborated on an equal level.

\section{References}

1 Barr J, Fraser GL, Puntillo K, Ely EW, Gélinas C, Dasta JF, et al.; American College of Critical Care Medicine. Clinical practice guidelines for the management of pain, agitation, and delirium in adult patients in the intensive care unit. Crit Care Med. 2013 Jan;41(1):263-306.

2 Reade MC, Finfer S. Sedation and delirium in the intensive care unit. N Engl J Med. 2014 Jan;370(5):444-54.

3 Acevedo-Nuevo M, González-Gil MT, Romera-Ortega MÁ, Latorre-Marco I, Rodríguez-Huerta MD. The early diagnosis and management of mixed delirium in a patient placed on ECMO and with difficult sedation: A case report. Intensive Crit Care Nurs. 2018 Feb;44:110-4.

4 DeGrado JR, Hohlfelder B, Ritchie BM, Anger KE, Reardon DP, Weinhouse GL. Evaluation of sedatives, analgesics, and neuromuscular blocking agents in adults receiving extracorporeal membrane oxygenation. J Crit Care. 2017 Feb;37:1-6. 
5 deBacker J, Tamberg E, Munshi L, Burry L, Fan E, Mehta S. Sedation Practice in Extracorporeal Membrane Oxygenation-Treated Patients with Acute Respiratory Distress Syndrome: A Retrospective Study. ASAIO J. 2018 Jul/Aug;64(4):544-51.

6 van den Boogaard M, Schoonhoven L, Evers AWN, van der Hoeven JG, van Achterberg T, Pickkers P. Delirium in critically ill patients: impact in long-term health-related quality of life and cognitive functioning. Crit Care Med. 2012 Jan;40(1):112-8.

7 Palencia-Herrejón E, Romera MA, Silva JA; Grupo de Trabajo de Analgesia y Sedación de la Semicyuc. Delirio en el paciente crítico. Med Intensiva. 2008;32(Suppl. 1):77-91.

8 Christensen M. An exploratory study of staff nurses' knowledge of delirium in the medical ICU: an Asian perspective. Intensive Crit Care Nurs. 2014 Feb;30(1):54-60.

9 Barnes-Daly MA, Phillips G, Ely W. Improving hospital survival and reducing brain dysfuntion at seven California Community Hospitals: implementing PAD guidelines via the ABCDEF bundle in 6,064 patients. Crit Care Med. 2017 Feb;45(2):171-8. 